What is a crystal to the new chemical crystallographer, after that first, automated structure analysis?

Larry R. Falvello

Department of Inorganic Chemistry and Aragón Materials Science Institute, University of Zaragoza - CSIC, Zaragoza, Spain

Pedro Cerbuna 12, E-50009 Zaragoza, Spain. Email: falvello@unizar.es

ORCID: 0000-0002-0444-996X

Researcher ID: M-1499-2014

Facebook: Lab Larry Falvello 


\section{What is a crystal to the new chemical crystallographer, after that first, automated structure analysis?}

This educational review postulates the importance of maintaining an adequate level of crystallographic education among structure-dependent scientists whose interests are not primarily in crystallography, at a time when automation and validation have made it possible to obtain high-quality structure analyses in many cases with a minimum of crystallographic background. The topics addressed are intended to form a second round of crystallographic education for a novice user whose first round involved hands-on experience with structure solution and an introduction to elementary concepts. The specific topics, chosen for their relevance as basic knowledge and their lack of emphasis in many formal treatments, are 1) crystallographic reference frames and the utility of the reciprocal cell in geometrical calculations; 2) the relationship between the two concepts that constitute our model of the crystal, namely the unit cell and the lattice; 3 ) the manner in which an atom is represented in concept and in practice; 4) the importance of interleaved symmetry elements required by the presence of additional symmetry on a lattice; 5) the harnessing of the natural properties of the crystalline state for the potential manipulation of properties of synthetic crystals; and 6) useful terminology for navigating a crystal structure.

Keywords: crystallographic education; crystallographic reference frames; reciprocal lattice; atomic scattering factor; crystallographic symmetry; crystal engineering 


\section{Contents}

1. Introduction 3

2. We use a skew-angular reference frame to describe what is in a 7

3. A crystal is described as having two conceptual parts, a discrete 11 lattice and a continuous pattern.

4. An atom within a crystal has an easily understood representation. $\quad 15$

$\begin{array}{ll}\text { 4.1. Why would we care about the details of this? } & 17\end{array}$

5. A crystal seems to add to its own symmetry. 18

6. A crystal is synthetic -- or is it natural? 25

7. A crystal is a set of addressable points, which can be described by 27 atom designator codes.

8. Closing Comments. 30

Acknowledgments

Notes on contributor

References

\section{Introduction}

The IUCr Online Dictionary of Crystallography would seem an appropriate place to look for an authoritative definition of a crystal [1]. But as related by Chapuis \& Brock in the IUCr Newsletter [2], in deliberations about the Online Dictionary, '... a recurring point of discussion concerns the definition of a crystal.' For the record, the definition in the Online Dictionary at the time of this writing is, 'A material is a crystal if it has essentially a sharp diffraction pattern.' ('Essentially' is defined subsequently.)

Approaching the nature of crystals from a slightly different direction, namely an on-line search engine, we might find subjective references to crystals in "stunning earrings and diamond rings," but we can also read about more functional crystal uses such 
as "solar-powered devices," "solving important biological puzzles," "monitors for computers," and even "tasty snacks." At the site that describes the latter applications [3], we find the definition of a crystal as "any solid material with its atoms, or smallest units of matter, organized in a repeating pattern."

The focus of that definition is on the crystal as a self-standing item, as opposed to the way it responds to a probe.

Elsewhere, continuing to search on line, one can find information aplenty about the purported healing powers of crystals, with a lesser amount of effort devoted to questioning such claims [4].

And there is much more. But one conclusion that emerges from the abundant information to be found about crystals, is that the definition of a crystal depends upon the context in which it is being conceived and upon who is doing the defining.

One scientific figure that has emerged in substantial numbers and has evolved over the last half-century is the user of crystallography, a scientist who may or may not be an expert crystallographer but who applies diffraction to the solution of chemical problems, often beginning with the identification of new products. One factor that promoted the application of crystallography to chemical analysis, long before anyone promulgated the thought that a million and more unique substances would one day be thus characterized, was the realization that the alternative approach using spectroscopy, physical measurements and analysis was more difficult and time-consuming. As pointed out by Cotton \& Troup [5], the identities of products from a complex preparation could be unraveled by x-ray crystallography, which they described as 'a practical means of cheap, rapid and definitive analysis.' The same article described their decision to use xray analysis first for a given product, instead of beginning with laborious alternative forms of characterization, as a 'tactical approach employed which, we think, is both novel and 
uniquely powerful.' This was one prominent example of diffraction analysis being given more protagonism in chemical research, but it was not the only one. In the ensuing years the use of x-ray structure analysis in chemistry has reached massive proportions, to the extent that informally the existence of a new compound in some areas is not accredited without a crystal structure analysis.

And that has led to a situation whose nature is hidden in plain view: Crystals and crystallography are very useful -- indeed powerfully important -- to a large number of scientists whose scientific interests and goals are not focused on crystals and crystallography as such. Moreover, automation now makes it possible to conduct a routine structure analysis with little or no knowledge of the fundamental principles of crystallography or of the internal workings of the procedures employed. Quality control, which in crystallography is called structure validation, serves to identify possible problems in the results, as a further aid in assuring a successful outcome [6,7].

The increasing use of diffraction crystallography by scientists whose interests and expertise lie elsewhere may be concerning to those who have studied crystallography in depth and who may worry about things like the continued use of proper terminology. This begs the question of what constitutes an appropriate level of crystallographic education for the chemist for whom crystal structure serves as an accessory to research whose focus lies in other areas.

There have been many good books written about crystallography and about the use of diffraction in analyzing crystal structures. These have excellent discussions of technique as it existed at the time the books were written. More recent books continue in that trend; and one can find modern texts with both in-depth and practical coverage of crystallography and structure analysis $[8,9,10]$. With an eye to the future, and recognizing that an in-depth understanding of crystallography will not be a goal for everyone who 
uses crystallography in chemical research, it will still be useful to point out a few features of crystals and their study which the structural chemist really ought to know -- topics which may have received treatment in many of the books that have dealt with crystallography, but which in the future may not reach the non-crystallographer who uses crystallography.

The text that follows presents selected topics that the author considers important as elements of fundamental knowledge of crystals and crystallography, but which are not of daily concern to users. These concepts -- or at least the way they are presented here -are features of crystals and crystallography that have not always received explicit attention, and which will be useful to, but may not be noticed by, chemists who use crystallography to good effect without actually being interested enough in crystallography per se to study it in depth.

Knowledge of some preliminary concepts is assumed; that knowledge is acquired nowadays in a first experience or experiences with structure analysis, and consists of what one ought to have learned when conducting those first analyses..This includes fractional crystallographic coordinates, basic symmetry concepts, crystal systems, and a minimal exposure to the idea of the reciprocal lattice. The present article is an attempt to convey the fundamental knowledge that should come next -- something of a 'Part Two' in basic crystallographic training. Some mathematical constructs are assumed to be familiar -- the Fourier transform and the delta function. Convolution will be mentioned to the extent that it helps us understand crystals; but it will not be described in depth.

The treatment begins with crystallographic reference frames as they apply to geometrical calculations. The differences between these and the more familiar Cartesian system of reference are important. A fact that receives little attention now that computers 
do all of this work, is that the reciprocal cell makes an appearance here as a necessary if not always explicit partner in the calculations.

From there we continue with details of just what constitutes an atomic site in our model of a crystal structure. Some mathematics will intervene, but in quite tractable terms. Knowledge of how atoms are actually represented in structures is likely to become important to the structural chemist, as alternative ideas for more accurate representations of atoms are getting closer to implementation for routine analysis.

The final principal point deals with symmetry and closure. Any user of crystal structure analysis, whether an active analyst or someone who extracts results from a data base, will encounter crystallographic symmetry as a necessary instrument for examining the results. The effects of closure (group closure, that is) on the distribution of symmetry elements in a periodic structure will be described in the most basic terms.

The article continues with commentary on the preponderance of synthetic crystals in structure analysis and what we might derive from studying them. Some closing comments are offered on symmetry-related terminology defined in the early days of crystallographic computing but which is still useful today.

\section{We use a skew-angular reference frame to describe what is in a crystal.}

Many geometrical calculations involving crystals, the atoms that compose them, and the diffraction that they produce, can be most conveniently described in non-Cartesian coordinate systems. There are many possible ways to connect the dots that represent a lattice [Figure 1(a)], and equally many ways to define the basis vectors $\boldsymbol{a}, \boldsymbol{b}$ and $\boldsymbol{c}$ and the corresponding scalar unit-cell parameters $a, b, c, \alpha, \beta$ and $\gamma$. The shape of the unit cell, and the corresponding basis vectors of the lattice for a given crystal, are defined by the user or by a computer program according to crystallographic convention as established 
for each of the crystal systems. In simpler terms, nature gives us unit cells whose axes are not necessarily perpendicular to each other and which are not necessarily the same length.

The concept of fractional crystallographic coordinates is assumed to be understood and will not be treated in depth here. A point within the unit cell, an atom for example, is located at coordinates (xyz), corresponding to displacement from the origin by a fraction $x$ of the $\boldsymbol{a}$-axis repeat, a fraction $y$ of the $\boldsymbol{b}$-axis repeat and a fraction $z$ of the $\boldsymbol{c}$-axis repeat. The non-Cartesian frame of reference, whose unit repeats are the $\boldsymbol{a}$-, $\boldsymbol{b}$ - and $\boldsymbol{c}$-axes of the unit cell, is called a skew-angular reference frame [Figure 1(b)].

The use of skew-angular reference frames does not make our work, or the work of computer programmers, any more difficult in practice. On the contrary, some things such as the expression of symmetry operations, are considerably simpler when a natural reference frame is used. But it is necessary to expand some of our basic concepts and calculations to accommodate the non-orthogonal nature of the basis vectors.

The omnipresent feature of these reference frames is that they come in pairs. A skew-angular reference frame has a complement, called its adjoint reference frame. For any crystallographic reference frame based on the unit cell $\boldsymbol{a}, \boldsymbol{b}, \boldsymbol{c}$, its adjoint is, as it materializes, the corresponding reciprocal cell $\boldsymbol{a}^{*}, \boldsymbol{b}^{*}, \boldsymbol{c}^{*}$. It should not come as a disappointment to a practicing crystallographer, that reciprocal space, as we call it, is not the exclusive domain of crystallography but rather is also part of the more general and more generally useful concept of skew-angular, adjoint reference frames.

[As Sands has written [11], 'The notion of reciprocal space is not the exclusive domain of crystallographers, however, although they are inclined to be possessive about it.' To be fair, it should be noted that solid state physics also makes extensive use of the concept of reciprocal space.] 
A thorough description of skew-angular reference frames is given by Lanczos [12], with the adjoint defined in the same fashion in which we define our reciprocal cell, through its orthogonality and reciprocal relationships with the direct reference frame [equation (1)].

$$
\begin{array}{lll}
\boldsymbol{a} \cdot \boldsymbol{a}^{*}=1 & \boldsymbol{a} \cdot \boldsymbol{b}^{*}=0 & \boldsymbol{a} \cdot \boldsymbol{c}^{*}=0 \\
\boldsymbol{b} \cdot \boldsymbol{a}^{*}=0 & \boldsymbol{b} \cdot \boldsymbol{b}^{*}=1 & \boldsymbol{b} \cdot \boldsymbol{c}^{*}=0 \\
\boldsymbol{c} \cdot \boldsymbol{a}^{*}=0 & \boldsymbol{c} \cdot \boldsymbol{b}^{*}=0 & \boldsymbol{c} \cdot \boldsymbol{c}^{*}=1
\end{array}
$$

How does this affect our calculations? We use the scalar product (dot product) of two vectors, or of a vector with itself, in several critical calculations -- among them the calculation of interatomic distances.

Suppose that we have an interatomic vector -- describing a chemical bond, for example -- whose coordinates are $\Delta x, \Delta y$ and $\Delta z$. The length, $d$, of this vector is the square root of its scalar product with itself [equation (2)]. When using non-orthonormal coordinates, what will now be different from the Cartesian case is the manner in which the scalar product is calculated. For a skew-angular system, one of the two vectors has to be expressed in the 'direct' (crystallographic) reference frame and one has to be expressed in the adjoint (reciprocal) reference frame [equation (3)]. If $(\Delta x, \Delta y, \Delta z)$ are the coordinates of the vector in direct space and $(\Delta u, \Delta v, \Delta w)$ are the coordinates of the same vector expressed in terms of the reciprocal axes, then the dot product of this vector with itself is given simply as in equation (3).

$$
\begin{gathered}
d^{2}=\boldsymbol{d} \cdot \boldsymbol{d} \\
d^{2}=(\Delta x, \Delta y, \Delta z) \cdot(\Delta u, \Delta v, \Delta w)=\Delta x \Delta u+\Delta y \Delta v+\Delta z \Delta w
\end{gathered}
$$

But if we have only the coordinates of a vector -- an interatomic vector, say -- in direct, crystal space, how do we determine the coordinates of the same vector on the 
reciprocal basis? This is done by pre-multiplying the real-space vector by the metric tensor of the reference frame. The metric tensor, also known by other names, has several functions in crystallography; but in the present context it is the means of measuring things in our reference frame [equations (4) and (5)].

$$
\begin{aligned}
& \left(\begin{array}{lll}
\Delta u & \Delta v & \Delta w
\end{array}\right)=\left[\begin{array}{lll}
\boldsymbol{a} \cdot \boldsymbol{a} & \boldsymbol{a} \cdot \boldsymbol{b} & \boldsymbol{a} \cdot \boldsymbol{c} \\
\boldsymbol{b} \cdot \boldsymbol{a} & \boldsymbol{b} \cdot \boldsymbol{b} & \boldsymbol{b} \cdot \boldsymbol{c} \\
\boldsymbol{c} \cdot \boldsymbol{a} & \boldsymbol{c} \cdot \boldsymbol{b} & \boldsymbol{c} \cdot \boldsymbol{c}
\end{array}\right]\left(\begin{array}{l}
\Delta x \\
\Delta y \\
\Delta z
\end{array}\right) \\
& d^{2}=\left(\begin{array}{lll}
\Delta x & \Delta y & \Delta z
\end{array}\right)\left[\begin{array}{lll}
\boldsymbol{a} \cdot \boldsymbol{a} & \boldsymbol{a} \cdot \boldsymbol{b} & \boldsymbol{a} \cdot \boldsymbol{c} \\
\boldsymbol{b} \cdot \boldsymbol{a} & \boldsymbol{b} \cdot \boldsymbol{b} & \boldsymbol{b} \cdot \boldsymbol{c} \\
\boldsymbol{c} \cdot \boldsymbol{a} & \boldsymbol{c} \cdot \boldsymbol{b} & \boldsymbol{c} \cdot \boldsymbol{c}
\end{array}\right]\left(\begin{array}{l}
\Delta x \\
\Delta y \\
\Delta z
\end{array}\right)
\end{aligned}
$$

If we expand the scalar products in the metric tensor, equation (5) becomes the basis of the scalar equation that we can find in textbooks [equations (6), (7)].

$$
\begin{gathered}
d^{2}=\left(\begin{array}{lll}
\Delta x & \Delta y & \Delta z
\end{array}\right)\left[\begin{array}{ccc}
a^{2} & a b \cos \gamma & a c \cos \beta \\
a b \cos \gamma & b^{2} & b c \cos \alpha \\
a c \cos \beta & b c \cos \alpha & c^{2}
\end{array}\right]\left(\begin{array}{l}
\Delta x \\
\Delta y \\
\Delta z
\end{array}\right) \\
d^{2}=a^{2}(\Delta x)^{2}+b^{2}(\Delta y)^{2}+c^{2}(\Delta z)^{2}+2 a b \cos \gamma \Delta x \Delta y+2 a c \cos \beta \Delta x \Delta z \\
+2 b c \cos \alpha \Delta y \Delta z
\end{gathered}
$$

Classic texts such as Stout and Jensen [13] give Equation (7) or a similar expression as the bond-length calculator for triclinic crystals. When the symmetry is higher, terms begin to vanish from the metric tensor or to become equal to each other; and the expression becomes simpler. For example, for a monoclinic crystal with $\boldsymbol{b}$ unique, the angles $\alpha$ and $\gamma$ are $90^{\circ}$ and so the terms containing $\cos \alpha$ and $\cos \gamma$ vanish.

Things work just the same in the reverse direction. The reciprocal metric tensor can be placed between two vectors, given in reciprocal coordinates -- for example between $(h k l)$ and itself, to calculate the scalar product. Thus, the length $\left|d^{*}\right|$ of a reciprocal lattice vector can be calculated as a first step in computing $2 \theta$ for the reflection (hkl), since $\left|d^{*}\right|=2 \sin \theta / \lambda$ ( $\theta$ and $\lambda$ are the Bragg angle and the wavelength). This is also 
an easy way to calculate the spacing $d(h k \ell)$ between Bragg planes, since $d(h k \ell)$ is the reciprocal of $\left|d^{*}(h k \ell)\right|$. Such calculations can easily be programmed in a spreadsheet application.

There is more to the subject of skew-angular reference frames in crystallography, but for now we will simply comment that geometry calculations really are no more complicated in principle than they are in Cartesian reference frames, for which the metric tensor appears as a unit, diagonal matrix and is thus usually not shown, and for which a given point has the same coordinates in both the real and reciprocal reference frames.

\section{A crystal is described as having two conceptual parts, a discrete lattice and a continuous pattern.}

We shall adhere for present purposes to the long-used idea of a crystal as consisting of identical unit cells repeated in all directions. In recent decades an extension of this picture of the crystal, able to account also for incommensurate modulations, incommensurate composites and quasicrystals, has been necessary. In the context of chemical crystallography, we more often than not deal with crystals that can be treated as periodically homogeneous substances, in which a pattern formed principally by atoms is repeated in regular intervals in all directions [14]. This breaks our formal view of the crystal down into two component concepts - the pattern and the lattice. The basic pattern in this deconstruction is the set of objects -- usually modelled as atoms although more nuanced treatments can be conceived -- bounded by the unit cell, a parallelepiped of general shape. The contents of the unit cell constitute our continuous pattern, even if these contents are granular, consisting of atoms rather than a smoothly varying mass. It is important, in a formal development, to keep sight of the fact that in our periodically homogeneous model the unit cell is a useful conceptual boundary around our continuous pattern of objects; the unit cell itself is not a physical object. Neither is the 
lattice, which is a pattern of repetition by translation. The result is our traditional description of a crystal, which has the unit cell contents being repeated along the three defining edges of the cell, whose dimensions and relative orientations thus correspond to the basic axes of the lattice.

The unit cell contents, being physical objects, are easy to represent. We can draw a picture depicting all of the atoms that have been found in one unit cell during a structure analysis, along with any other feature that has been inferred by us from the arrangement of atoms. The most-often inferred feature is the chemical bond. These do not emerge directly from structure analyses conducted in the usual manner, with spherical-atom scattering factors, but rather are drawn by the user or by a computer program based on chemical knowledge in the form of tabulated atomic radii or compilations derived from data bases.

The lattice, in contrast, is not a tangible physical object but rather a description of the translational periodicity relating one unit cell to the others. We usually think of it as three translations represented as vectors, and indeed its basis is just that. But the lattice is the entire pattern, and there are certain contexts in which it is useful to remember that lattice translational symmetry applies to any combination of integer multiples of those three basis vectors. So for example, the translation [111], which is the vector sum of $1 \boldsymbol{a}$ $+1 \boldsymbol{b}+1 \boldsymbol{c}$, is also a lattice vector which embodies a translational symmetry relationship between whatever is at its starting point, the vector tail, and whatever is at its end, the head of the vector.

It should be remembered that representations of the lattice are representations and not physical objects. We can represent the lattice by a set of intersecting lines whose points of intersection are connected by lattice translations. The lines also delimit the unit- 
cell parallelepipeds. Another often-seen representation of the lattice is a set of dots in which the travel from one dot to any other is a lattice translation.

The division of the crystal into these two conceptual components permits a simple mathematical description of the crystal as the convolution of the unit cell with the lattice.

In order to effect that deconstruction of the crystal, we invoke simple mathematical descriptions of the unit-cell contents -- e.g., atomic coordinates with respect to the unit-cell axes -- and of the basis vectors of the lattice. The crystal is a single object, but as has been mentioned earlier with reference to Figure 1(a), there exist any number of ways to choose a basis set for the lattice and thus the corresponding unitcell dimensions. The following treatment assumes that such a choice has been made, so that the basis sets for the lattice vectors and for coordinates within a unit cell have been established. (In practice, the choice is made, usually by a computer program, in conformity with established crystallographic conventions.)

A mathematical description of the crystal comes in handy when we want to understand the formulas that we use for analyzing structures. And the math is not too complex, even if the initially daunting operation of convolution is involved. For now we shall regard the contents of one unit cell as a continuous function that describes the electron density (or any other scattering density) as a function of position within the basic parallelepiped. That is, for each point with fractional coordinates $(x y z)$ there is a function with a value of $\rho(x y z)$-- also referred to as $\rho(\boldsymbol{r})$, in which $\boldsymbol{r}$ is the vector from the origin to the point $(x y z)$.

The lattice also has a mathematical description, as an unbounded array of (Dirac) delta functions -- that is, it has a value of 1 (one) at lattice points and a value of zero everywhere else. 
For a mathematical representation of the crystal as a combination of the unit cell and the lattice, it is necessary to invoke convolution. Fortunately, a convolution involving a delta function (the lattice) with a continuous function that has the same dimensions as the lattice (the unit-cell contents) simply repeats the continuous function at each of the lattice points. Thus we have the crystal as a periodically homogeneous entity -- a neat and neatly manipulable mathematical representation of our unit cell and its convolution with the lattice.

A rigorous derivation of this mathematical representation of the crystal can be found in Woolfson [15]. This and other text books then invoke the convolution theorem to describe the diffraction pattern, which is the Fourier transform of the crystal. Here we use lowercase letters for the original functions and uppercase for the Fourier transforms. We use $x$ for the domain of the original functions (for us, the crystal) and $S$ is the domain of the Fourier transforms (the diffraction pattern). According to the convolution theorem, if we have the convolution $c(x)$ of two mathematical functions [equation (8)], then the Fourier transform of $c(x)$, called $C(S)$, is the product -- not the convolution -- of the two Fourier transforms $F(S)$ and $G(S)(9)$.

$$
\begin{gathered}
c(x)=f(x) * g(x)=\int_{-\infty}^{\infty} f(u) g(x-u) d u=g(x) * f(x) \\
C(S)=F(S) G(S)
\end{gathered}
$$

In our view of the crystal, what is inside the unit cell, its full contents, is $f(x)$, the lattice is $g(x)$ and the crystal is $c(x)$. The diffraction pattern $C(S)$ is the Fourier transform of the crystal, so it is the Fourier transform $F(S)$ of the unit cell, times the Fourier transform $G(S)$ of the lattice.

The whole description gets much simpler because the lattice is a set of delta functions. The Fourier transform of the unit cell is a continuous function in reciprocal space, and is called the structure factor. Its domain is defined by reciprocal coordinates 
$h k \ell$, so we usually see the structure factor represented as $F_{h k \ell}$. Although the domain of the structure factor is continuous, and values can be calculated for any $h k \ell$, whether integer or not, the fact that the diffraction pattern is the product of this function and the reciprocal lattice, which is a delta function, means that in actual measurements the diffraction pattern, as we know and use it, can only be sampled at points of the reciprocal lattice that have integer indices $h k \ell$.

What is actually measured is the intensity at each reciprocal lattice point; this intensity is related to the square of the magnitude of the structure factor, namely|$\left|F_{h k \ell}\right|^{2}$. This array of reciprocal lattice points with integer coordinates (indices), each with its own intensity, is called the intensity-weighted reciprocal lattice.

The structure factor, being in its full extent the Fourier transform of the contents of one unit cell, is the bridge between the diffraction pattern that is measured and the structure that we are trying to analyse. It will be this set of structure factor magnitudes, $\left|F_{h k \ell}\right|$, or their squares, that we will use as data in deriving the parameters that define the structure -- element types, atomic coordinates and displacement parameters.

The Fourier transform of a single delta function located at the origin of a coordinate system is a constant function with a value of 1 . When that delta function is extended into an unbounded lattice-like array of delta functions -- that is, with a value of 1 at any lattice point and a value of zero everywhere else -- its Fourier transform is another array of delta functions for which the value of the function is 1 at reciprocal lattice points and zero everywhere else.

\section{An atom within a crystal has an easily understood representation.}

The structural chemist does not usually worry about the mathematical representation of atoms in structure analysis. Atoms appear in pictures on a screen, seemingly out of the clear blue - usually out of a structure solution program in the first 
instance - and for those atoms that fit into an acceptable pattern with the others, renaming or reassigning to a different element ( $\mathrm{C}$ for $\mathrm{N}$, etc.) is the most that the user has to do. Disorder may require further adjustments, such as to the occupancy. Spurious atoms are deleted. But even then, the manner in which atoms enter into our calculations is a background function. We have to be concerned only with the veracity of three atomic properties - assignment to an element, the position of the atom in the unit cell and the displacement parameters.

The budding structural chemist may see the structure factor equation in class or in a text book, but in practice this equation is a silent partner whose inclusion in the calculations is almost invisible to the user. In this equation $(10,11)$, the element assignment appears in the form of the atomic scattering factor, $f_{j}$. The displacement parameters $U^{i j}$ appear in the exponent of a term that attenuates the scattering factor, and the atomic coordinates appear in the exponent of the phase factor (the second exponential in equation (10).

$$
\begin{gathered}
F_{h k \ell}=\sum_{\text {atoms } j} f_{j} \exp ^{-T} \exp ^{2 \pi i\left(h x_{j}+k y_{j}+\ell z_{j}\right)} \\
T=2 \pi i\left(h^{2} a^{* 2} U^{11}+k^{2} b^{* 2} U^{22}+\ell^{2} c^{* 2} U^{33}+2 h k a^{*} b^{*} U^{12}+2 h \ell a^{*} c^{*} U^{13}\right. \\
+2 k \ell b^{*} c^{*} U^{23}
\end{gathered}
$$

The expression inside the summation in Equation (10) is the wave scattered by atom $j$ in the direction of the scattered beam for reflection $h k \ell$. The atomic scattering factor for atom $j$, namely $f_{j}$, in its simplest form (ignoring resonant scattering) is a scalar and is the amplitude scattered by an at-rest atom of the element assigned to $j$, for the scattering angle $2 \theta$ of reflection $h k \ell$. This amplitude is diminished by the first exponential term, $\exp ^{-T}$, which gives the attenuation resulting from the motion of the atom in the direction of the scattering vector for $h k \ell$. The degree of attenuation depends on the length of the scattering vector and on the magnitudes of the displacement parameters $U^{i j}$. The 
$U^{i j}$ are variable parameters pertinent to a given atom; their values emerge from structure refinement and can be used to calculate the mean-square displacement of the atom in any direction.

The point that we are getting at here, though, is this: If the scattering from an atom -- that is, its footprint in reciprocal space -- is described as the product of two functions, namely the scattering factor $f_{j}$ of the atom at rest and the displacement attenuation $\exp ^{-T}$ resulting from atomic motion and other possible factors, then our model for the atom itself -- in direct or crystal space -- must be the convolution of the two corresponding Fourier transforms. The Fourier transform of the displacement-caused attenuation is the probability density function for the atom in question; this function gives the probability of finding the center of the atom at any location displaced from the mean position described by the atomic coordinates $(x y z)$. And the Fourier transform of the at-rest atomic scattering factor is the electron density of the atom in crystal space. The probability density function that convolves the electron density has the effect of smearing the at-rest electron density according to the probabilities of each of the possible finite displacements of the atom from its mean position. The cumulative effect of that smearing is usually aphorized graphically in the form of a displacement ellipsoid.

A full derivation of this 'convolution approximation' is given by Dunitz [16], who also gives useful background information on the mathematical operation of convolution.

4.1. Why would we care about the details of this? The question arises naturally, because crystallographers, chemists and other scientists have been analyzing crystal structures for many decades without often entering into the details of atomic scattering factors. And even the displacement parameters, while usually needed for a refinement to be considered successful, have been treated as necessary accessories but have often been given short shrift when it comes to analyzing the results of a structure determination. 
It turns out that there are credible alternative ideas regarding atomic scattering factors. Those that we have been using for the past half-century or more are based on accurate electronic-structure calculations for neutral, spherical atoms of the different elements [17]. These have been fit for purpose in structure analysis as usually conducted. However, the availability of more accurate diffraction data, better resolution, and vastly improved computing facilities -- as compared to conditions as they existed when those scattering factors were first employed -- have made it realistic to consider using more accurate and contextual representations of electron density, especially valence electron density.

Non-spherical scattering factors for valence electron density, in the form of expansions of multipole functions, have been used for some time [18], but have remained in the category of specialized applications. These are purely mathematical representations, but the Fourier invariance of the multipole functions facilitates the interpretation of the results in terms of orbital-like representations. Macchi et al. [19] describe this method and also briefly summarize other credible ideas for determining aspherical electron density using diffraction.

In a more recent development, the so-called Hirshfeld atom refinement [20], quantum chemistry calculations are employed in what amounts to a contextual extension of the spherical-atom model to include the electron density of an atom as it exists in the crystal. The molecular electron density emerging from the calculation, which can conveniently be a DFT calculation but in principle could be any reliable quantum chemistry procedure, is partitioned into atoms using the Hirshfeld 'stockholder' scheme [21]; and the scattering factors for these new atomic descriptions are calculated for use in the next round of refinement. 
Scattering factors for non-spherical electron density are beginning to appear in the most widely popular software for chemical structure analysis [22]. The methods have been tested -- in the case of multipole expansion refinement, for decades -- and the modern implementations will bring these within reach of scientists whose interest is in the electron density more than in the diffraction. It is important that users of these techniques have at least a basic understanding of how an atom in a crystal is represented.

\section{A crystal seems to add to its own symmetry.}

As mentioned earlier, crystallography is very useful to many scientists who are not primarily interested in crystals. In addition to the occasional complaint by experts about misused terminology or poor refinement technique, there arises the more fundamental question of crystallographic education for those non-crystallographers who nevertheless are users and/or consumers of crystallographic results.

There are some skills and topics whose need has been irreversibly supplanted by technological advances, including but not limited to advances in software. Weissenberg photography is an example, along with no end of computing skills such as the ability to write formatted input files for old programs. But there are other items whose evident loss of importance is more illusory. Education in crystallographic symmetry is the most important of these. Software advances have made it possible to solve and refine many crystal structures without more than a passing idea of the nature and consequences of space group symmetry. It has been quite some time now since the use of extinction symbols for determining the possible space groups for a given structure became a software function and exempted the user from the need to know how to do it. Another important barrier has fallen in recent years, now that there are structure solution programs that can solve many structures without recurring in the first instance to space group symmetry. 
Unlike the ingenious devices created by crystallographic pioneers -- the Weissenberg camera, or Beevers-Lipson strips, for example -- symmetry is an intrinsic property of crystals; and in the author's opinion it is a subject that merits attention from all users of crystallographic results.

There is one important aspect of crystallographic symmetry that has not usually been given special attention, namely the consequences of closure and the presence of inequivalent symmetry elements. These are the subject of this section.

We will begin with a monoclinic example, by way of demonstrating the effects of closure. A newcomer to crystallographic symmetry will notice that the graphical representation of a simple monoclinic space group, for example P2 (Figure 2) appears to have more symmetry elements than those that are listed explicitly in the International Tables. For $\mathrm{P} 2$, with the $\boldsymbol{b}$-axis unique, the listed symmetry consists of the unitary operation $(x, y, z)$ and the two-fold axis, $(-x, y,-z)$. This covers a representative point [labelled 1 in Figure 3(a)] and its congener related by the two-fold axis (point $1 \mathrm{~A}$ in the figure). These two are related by the two-fold symmetry axis labelled 1 in red. If we now operate on point $1 \mathrm{~A}$ with a lattice translation parallel to the $\boldsymbol{a}$-axis of the cell, the resulting equivalent position is 1B, Figure 3(b). The symmetry of a crystal -- including the translational symmetry described by the lattice -- forms a group, and thus must have the property of closure, meaning in this case that there has to be a symmetry operation that relates point 1 and point $1 \mathrm{~B}$. That operation is $(1-x, y,-z)$, which is what we get by operating first with the two-fold axis and then with the lattice repeat along the $\boldsymbol{a}$-axis.

What is this operation, qualitatively? It is not represented in the list of general equivalent positions (the two given above) for this space group. As it turns out, this is another two-fold symmetry axis, parallel to the first and displaced half-way along the lattice translation. This two-fold axis, '2' in red in the figure, is not equivalent to the first; 
that is, there is no symmetry operation of the space group that carries the first two-fold axis into this one. Yet the basic assumptions for deriving this simple monoclinic space group -- the presence of a two-fold axis parallel to $\boldsymbol{b}$ and a lattice translation perpendicular to that axis -- quickly lead to the necessary presence of this second symmetry element. As Azároff put it [23], this symmetry element 'springs up.'

Aside: This brings us to an important general consideration regarding crystallographic symmetry as used in chemical crystallography. We are accustomed to regarding the space group as a set of symmetry operations that fill out one unit-cell worth of content beginning with one asymmetric unit. A succinct definition of 'asymmetric unit' is given by Massa [24]:

'The asymmetric unit is the name given to that minimum group of atoms whose positions, together with those generated by the symmetry operations of the space group generate the complete contents of the unit cell.'

For space group $P 2$, the reference asymmetric unit (xyz) and its symmetry relative at $(-x, y,-z)$ give one cell's worth of contents (not all necessarily within the reference unitcell box). The lattice relates these to other unit cells. But the lattice is in reality part of the space symmetry of the crystal, and the consequences of this, namely group closure and the resulting presence of other, inequivalent symmetry operations, follow. These result from the interaction of a symmetry axis and a lattice translation perpendicular to that axis.

Needless to say, the result is general; so it will come as no surprise that the twofold axis along $\boldsymbol{b}$ and the lattice translation in the $\boldsymbol{c}$-direction also require the presence of an interleaved two-fold axis at $(0, y, 1 / 2)$, ' 3 ' in Figure 3(c). This axis relates points $1 \mathrm{~A}$ and $1 \mathrm{C}$ in the Figure.

And we are not finished. All lattice translations are relevant for the purpose at hand, and the face diagonal, [101], is also a lattice translation. So half-way along this 
displacement, there is another two-fold axis, labelled '4' in red in Figure 3(d). We can extend the analysis to longer lattice translations, but they will require the presence of twofold axes where we already have them. The picture is now complete; the symmetry of the crystal is closed.

Similar considerations hold for two-fold rotoinversion symmetry, which is in fact a mirror plane. To be clear about this operation, the mirror is associated with the direction perpendicular to the plane. We shall use the monoclinic space group Pm (b-axis unique) as our example. Its two listed symmetry elements are $(x y z)$ and $(x,-y, z)$. Operating on a point at (xyz), labelled '1' in Figure 4, with the operation $(x,-y, z)$, gives point $1 \mathrm{~A}$ in Figure 4. We further operate on 1A by the lattice translation [010], which is the operation $(x$, $1+y, z)$, and which carries point $1 \mathrm{~A}$ to $(x, 1-y, z), 1 \mathrm{C}$ in the figure. This is related to the initial point 1 by an interleaved mirror plane located at $y=0.5$. As with the proper twofold axis, this newly identified symmetry element is not related by symmetry to the original mirror at $y=0$. It is required by closure.

As has been described in detail by Buerger [25], the interaction of symmetry axes with lattice translations to require the presence of interleaved symmetry of the same order, is general and applies to all orders of crystallographic symmetry. It should be emphasized that in the case of a rotation axis, the relevant interaction is with a lattice translation perpendicular to the axis.

Buerger gives a rigorous analysis, and states, "...a rotation about an axis A through an angle $\alpha$, followed by a translation perpendicular to the axis, is equivalent to a rotation through the same angle $\alpha$, in the same sense, but about an axis B situated on the perpendicular bisector of $\mathrm{AA}^{\prime}$ and at a distance (AA'/2) $\cot (\alpha / 2)$ from $\mathrm{AA}^{\prime} . "$

$$
d=\left(\frac{A A^{\prime}}{2}\right) \cot \left(\frac{\alpha}{2}\right)
$$


Using Buerger's terminology, we return to the proper two-fold axis, whose rotation $\alpha$ is $180^{\circ}$, and its interaction with the lattice translation along the $\boldsymbol{a}$-axis of the cell. (So Buerger's AA' is the unit cell axis a.) The value of $\cot \left(90^{\circ}\right)$ is zero $\left(\cos 90^{\circ} / \sin 90^{\circ}\right)$. Thus, the interleaved two-fold axis lies at the midpoint of the lattice translation, and not displaced from it.

The situation is different when we consider a four-fold axis of symmetry. As before, we start with no assumptions other than the presence of a single four-fold axis [axis 1 in red, Figure 5(a)], a three-dimensional lattice, and the tetragonal unit-cell shape that is required by the presence of the symmetry element. Applying Equation 12, with $\alpha$ $=90^{\circ}, \mathrm{AA}^{\prime}=a=b$, i.e., $\mathrm{AA}^{\prime}$ is equal to the shortest lattice repeat in the $a b$-plane, and with $\cot \left(45^{\circ}\right)=1$, we find that another four-fold axis must be present, lying on the perpendicular bisector of the lattice repeat, and located $a / 2$ (or $b / 2$ ) from the lattice vector. That puts the additional four-fold axis in the middle of the unit cell, axis 2 in red in Figure 5(b).

And there is more. A four-fold rotation axis includes two-fold rotation symmetry as part of its fuller properties. (Or, the four-fold axis carries within it a two-fold axis; or the four-fold rotation, squared, is a two-fold rotation; or four-fold symmetry is also twofold symmetry -- however you prefer to describe it.) The presence of two-fold symmetry as part of the picture also requires the presence of the interleaved two-fold axes described above. So for any tetragonal crystal, its space group symmetry will exhibit the general pattern of Figure 5(c) -- the assumed four-fold axes separated by lattice translations, plus another, inequivalent four-fold axis in the middle of the pattern, and two-fold axes at the midpoints of the lattice vectors. N.b., the pattern has a different appearance when the fourfold symmetry is rotoinversion rather than pure rotation, and also for I-centered tetragonal space groups, but the principle is the same. See Buerger [25] for a full treatment. 
For a three-fold symmetry axis we shall use a hexagonal unit cell, Figure 6(a), with the symmetry element (axis 1 in the figure) at the corners of the cell. Equation 12 establishes the positions of our additional three-fold axes as those labelled 2 and 3 in Figure 6(b). The locations of these three-fold symmetry elements trisect the long diagonal of the $a b$-plane of the hexagonal cell. They are located on the perpendicular bisectors of the edges of the unit-cell base, at a distance of $a /(2 \sqrt{3})$ from their midpoints.

When we consider a six-fold axis interacting with a perpendicular lattice translation, Equation 12 requires the presence of a six-fold axis at a distance of $a \sqrt{3} / 2$ from the midpoint of the lattice translation $a$ (or $b$ ); and this coincides with the position of the original six-fold axis at a different corner of the unit cell. So there is no additional symmetry of order six. However, similarly to what pertained to the four-fold axis, the sixfold axis also has within it a three-fold axis and a two-fold axis -- which in operational terms are the square and cube of the six-fold symmetry. So the interleaved symmetry elements of orders two and three are present at their required positions, Figure 7.

A further consideration for crystals with six-fold symmetry is that, as with any crystallographic symmetry, the interleaved 3- and 2-fold symmetry can be proper or improper. Formally, a two-fold rotoinversion associated with the unit-cell $\boldsymbol{c}$-axis is a horizontal mirror plane -- that is, a mirror plane perpendicular to the $\boldsymbol{c}$-axis. These planes are also interleaved at half-cell intervals, as was described above for the monoclinic system.

The presence of interleaved two-fold symmetry associated with the unique symmetry direction in crystals with six-fold axes -- whether that two-fold symmetry appears as a rotation axis parallel to $\boldsymbol{c}$ or as a mirror plane or glide perpendicular to $\boldsymbol{c}$-provides a clear distinction between the symmetries classified as hexagonal and those classified as trigonal in the International Tables. Those symmetries listed as pertaining 
to the hexagonal crystal system have six-fold, three-fold and two-fold symmetry elements associated with the [001] direction, or $\boldsymbol{c}$-axis, of the hexagonal unit cell. Those symmetries classified as trigonal, when considered on the hexagonal cell, have only threefold symmetry associated with the $\boldsymbol{c}$-axis of that cell. The trigonal space groups as listed in the International Tables do not have any horizontal mirror symmetry. (In either of these two crystal systems, there can additionally be two-fold symmetry associated with directions perpendicular to the unique symmetry axis.)

In closing this section, we note that patterns of the sort represented in Figures 3-7 describe some of the plane-lattice types. In many treatments of crystallographic symmetry, the plane lattices, which are five in number, are used as a logical point of departure for describing the 14 three-dimensional lattice types as the results of stacking the two-dimensional lattices. We shall not undertake that derivation, which is well done in any number of excellent books and on-line treatments of the subject. We will note a few details. What we have described for monoclinic crystals, when taken only in the two dimensions represented in Figure 3, is a cell shape, namely a parallelogram, that can also be used when only unitary symmetry is present -- and thus can be stacked for describing either triclinic (without the two-fold symmetry) or monoclinic lattices. The common cell shape that we used in two dimensions for trigonal and hexagonal symmetries, the rhombus, is considered to be just one plane lattice type; it is used as a basis for the two lattice types ( $\mathrm{P}$ and $\mathrm{R}$ ) considered for trigonal crystals and the one, primitive lattice used for hexagonal crystals. The square lattice used above for tetragonal symmetry is the basis for deriving tetragonal and cubic lattices. There are, in addition, two rectangular plane lattices, which we have not considered, from which lattices are derived for orthorhombic crystals. 
The intended emphasis of this section, however, is the presence of interleaved symmetry elements that confer characteristic combinations of cell shapes and symmetry elements to the different crystal systems.

\section{A crystal is synthetic -- or is it natural?}

At the dawn of crystal structure determination and during its first decades, most structure analyses were conducted using naturally occurring inorganic crystals. With the techniques available at the time, the generally lower symmetries and larger asymmetric units of crystals of organic compounds rendered their structure analyses quite complex. In today's world of chemical research, molecular crystals of organic or metal-organic compounds are most often synthetic -- a synthetic compound isolated in crystalline form, or a natural compound that is not found in the crystalline state in nature. The possibilities that x-ray diffraction offered for the analysis of molecular structure using crystallized samples of organic molecules did not, however, escape the pioneers of the field. W. H. Bragg [26] (1921) wrote about the possibilities and provided data relevant to the structures of naphthalene and anthracene. The analysis of the structure of hexamethylenetetramine was the first definitive structure determination of an organic compound [27,28]. Graphite followed; and before the end of the decade of the 1920's, Lonsdale $[29,30]$ had established the structure of the aromatic ring in hexamethylbenzene.

These analyses accredited at least part of the optimistic comments by W. H. Bragg [26] about what could be done with knowledge of atomic positions in crystals:

'Moreover, the multiplicity of crystalline forms... each so precise and invariable, and so obviously related to the atomic and molecular forces, is a sign that if the forces were better understood it would be possible to account for the forms that are known, and possibly to build others that are unknown.' 
Bragg's comments appeared in an article titled 'The Structure of Organic Crystals.' Moving forward a century, with more than a million carbon-containing structures analyzed, it can be concluded that the lure of organic crystal structure analyses, expounded by the elder Bragg before a single organic structure had been definitively characterized, was real and has endured unabated.

W. H. Bragg's prescient idea about building previously unknown crystal forms would return as a broad cohort of strategies under the umbrella term 'crystal engineering,' defined by Desiraju [31] as 'the understanding of intermolecular interactions in the context of crystal packing and the utilization of such understanding in the design of new solids with desired physical and chemical properties.'

But despite the strong and growing efforts to manipulate the crystalline state itself, the use of synthetic crystals in structure determination has been principally aimed at chemical analysis. The continued development of x-ray diffraction to the point at which analyses are relatively simple to conduct has fomented its use as a supporting technique in synthetic chemistry. The crystal is a sample holder, keeping the target molecule in an ordered form that can produce the diffraction that is necessary for the analysis.

The two points of view of crystals were described by Hamilton \& Ibers [32]:

'From the first point of view, we look upon the crystal simply as a device for holding the molecule down so that we can take a good look at it. .... In the extreme of the second point of view... it is effects associated with the crystalline order which are of primary interest, and the molecule may be looked upon as an evil to be avoided if at all possible.'

In recent times, the role of the molecule is understood to include influencing the nature of the 'effects associated with the crystalline order.' 
The possibility of tuning the physical properties of molecular solids by deliberate adjustment of their compositions and/or structures, foreseen by W. H. Bragg, has maintained its attraction as a means of achieving useful functions, not least among them the efficient delivery of active pharmaceutical or agrochemical ingredients. This is an example of a natural order of things -- the forces that govern the formation and stability of crystals -- being harnessed for purposes not addressed by nature itself. As of this writing there have been some promising achievements; it is also clear that the broader field of crystal engineering is still developing, as the traditional structure-influencing features, hydrogen bonds and coordination geometries, are being complemented by the use of co-formers and by other interactions (halogen bonds, $\pi . . . \pi$ interactions and others) as possible tuning agents [33,34].

\section{A crystal is a set of addressable points, which can be described by atom designator codes.}

Upon looking through the results of a structure analysis, especially the geometrical parameters derived from the atomic positions, one will probably have encountered codes that look something like '1255501' or '1_555' or such. Crystallographers know how to use and manipulate these codes, but recent experience indicates that the newly minted structural chemist would be well served by a brief introduction. A description of the workings of these codes has been given by Spek [6].

The progenitor of all of these codes had a name and also a purpose that might not be obvious to the modern computer user. The name, 'atom designator code,' reflects the fact that this code designates a specific atom in an extended crystal structure -- that is, not one atom from the atoms list, but rather one member of the set of all equivalent atoms related to one atom in the atoms list. To specify the exact atom to which we are referring, we have to begin with an atom from the atoms list, then apply one symmetry operation 
from the list of symmetry operations, and then apply lattice displacements along $\boldsymbol{a}, \boldsymbol{b}$, or $\boldsymbol{c}$, or some combination of the three lattice repeats.

The atom designator code was defined in the original OR TEP manual [35] and can be found in the more recent version [36]. It is a packed number, and it simply contains the description of one atom -- atom number from the atoms list, symmetry operation to be applied to that atom, and lattice translations to be applied after the symmetry operation. As an example, suppose we have a structure with space group $P 2_{1} / n$, and we have the results of our refinement in a CIF.

The CIF will contain a loop with the symmetry operations in whatever order our refinement program listed them:

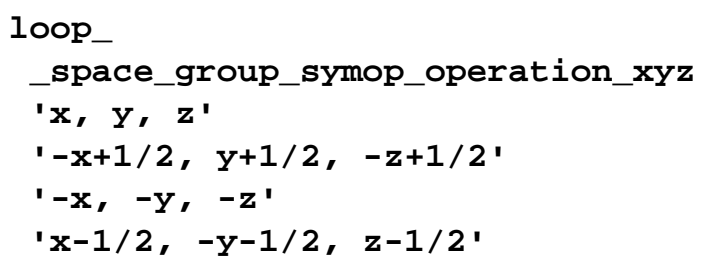

The CIF also includes the unit-cell parameters and a list of the atoms constituting one asymmetric unit (the reference asymmetric unit). In one recent example from the author's laboratory, in the geometry lists that were prepared by the refinement program, we find hydrogen bonds for which the acceptor atoms are related by symmetry to their congeners in the reference asymmetric unit. In the CIF the acceptor atom is given by name, for example 'O14,' and its site symmetry is given as 4_565. This means that the congener of $\mathrm{O} 14$ that accepts the hydrogen bond is the one that we get by starting with the coordinates of $\mathrm{O} 14$ from the atoms list (xyz), then applying symmetry operation 4 from the above list $(x-1 / 2,-y-1 / 2, z-1 / 2)$ and then applying a displacement of one lattice repeat parallel to the $\boldsymbol{b}$-axis to give $(x-1 / 2,-y+1 / 2, z-1 / 2)$. The code for the lattice translations, '565' uses 5 to mean zero, so that we can also use negative translations without creating any ambiguity. The three numbers correspond to the lattice translations 
to be applied parallel to the $\boldsymbol{a}$-, $\boldsymbol{b}$ - and $\boldsymbol{c}$-axes. A site-symmetry code of '2_645' for, let's say, atom $\mathrm{O} 12$, means that we begin with the coordinates of $\mathrm{O} 12$ as given in the atoms list -- call them $x, y, z$-- then apply symmetry operation 2 from the ordered list above -that brings us to $(-x+1 / 2, y+1 / 2,-z+1 / 2)$-- and then apply a displacement of +1 parallel to the $\boldsymbol{a}$-axis and -1 parallel to the $\boldsymbol{b}$-axis, giving us a symmetry relative of 012 located at $(-x+3 / 2, y-1 / 2,-z+1 / 2)$

The original atom designator code, as used by ORTEP, had all of the information in one number. The code '1255501' would mean atom 12 from the atoms list, with symmetry operation 1 applied ('01') and then lattice translations of zero for all axes ('555'). Lattice translations in the range -4 to +4 (rendered as 1 to 9 in the atom designator code) were allowed for all axes, so the user could describe atoms anywhere within a $9 \times 9 \times 9$ block of unit cells. That is, the contents of as many as 729 unit cells were hypothetically available for drawing.

For the purpose of terminology, the atom designator code (ADC) was distinguished from the structural object that it described. The atom described by the atom designator code was called the addressable point. These terms are still useful. The addressable point does not in fact have to be an atom. Any object to which coordinates can be assigned -- for example, the center of a ring -- can be an addressable point described by an atom designator code.

The atom designator code and the related concept of the addressable point provide a convenient shorthand description of a specific point in a structure. There was an additional reason that this construct was useful, as it permitted the program to store five items of information in a single integer storage location in the computer. In 1965 OR TEP could be run on a number of different computers that had $32 \mathrm{~K}$ words of memory. That can be compared to a typical desktop computer in use at the time of this writing, which 
would have something of the order of one-quarter million to a million times as much memory.

\section{Closing Comments}

As described above, in the model that is widely used to reproduce diffraction data, an atomic site is the convolution of a rest-atom scattering factor and a probability density function. We compile a list of atoms representing one asymmetric unit's worth of content, and together with the list of space group symmetry operations (implicitly and/or explicitly provided, depending on the program that we are using), this gives a description of the contents of one unit cell. The unit-cell dimensions, which also describe the lattice repeats, complete our usual description of the periodically homogeneous crystal; and when refinement has converged and there are no anomalous features, we consider the structure determination to have been completed.

This may be the point at which the crystallographer carves another notch. But for the structural chemist this may be the starting point of the analysis in chemical terms. For now, using the usual independent spherical-atom model that has been our mainstay, what we have is a list of atoms and the information needed to expand this list to include symmetry-equivalent atoms, to complete any incomplete molecules, to draw packing patterns, and so on.

Each (non-disordered) atomic site has three characteristics in our list -- its chemical element, its coordinates, and its displacement parameters. But in the end what we have are atoms. We have long delegated the drawing of bonds to computer programs, and up until the present the vast majority of bonds and non-covalent interactions that have been drawn or listed in structure analyses, by computer programs or explicitly inserted by the user, have been inferred to exist on the basis of geometrical data derived from the atom list and prior knowledge. As commented earlier, this may change in the foreseeable 
future; well-understood ideas for involving the diffraction data more directly in the characterization of bonding may be reaching a point of development at which they can be used more routinely.

Despite having accumulated a massive body of data from crystallographic analyses, we do not seem to be tiring of it. With technical developments continuing apace, it seems reasonable to foresee continuing use of diffraction crystallography for chemical analysis, with a natural extension to bonding as a component of the primary results. In addition, the knowledge base acquired from structural studies of crystals will continue to be used inductively, as W. H. Bragg put it nearly a century ago, 'to account for the forms that are known, and possibly to build others that are unknown.'

In all of these lines of development, it is necessary to work on the basis of a sufficient grounding in the concepts underlying our understanding of crystals and our use of physical techniques, including diffraction. The material presented in this piece represents a partial set of concepts that the author regards as important and easily overlooked.

\section{Acknowledgments}

Support was provided by the Ministerio de Ciencia, Innovación y Universidades (Spain, Grant PGC2018-093451-B-I00), the European Union Regional Development Fund, FEDER), and the Diputación General de Aragón, Project M4, E11_20R.

The author has no conflict of interest involving this work.

\section{Biographical Note}

Larry R. Falvello is Professor of Chemistry in the Department of Inorganic Chemistry at the University of Zaragoza and member of the Aragón Materials Science Institute, a joint institute of the University of Zaragoza and the Spanish Research Council (CSIC). He has been involved in the education and training of young crystallographers and chemists for some four decades, during which time the practice of crystallography, and the corresponding educational priorities for chemists, have undergone fundamental changes. His research group conduct their own syntheses, 
directed to the preparation of molecular crystals of coordination compounds with specific properties and transformations. He has served as Co-Editor (2002-2016) and Section Editor (2016 - present) at Acta Cryst., Section C and also as Associate Editor (2002-2014) at Comments on Inorganic Chemistry.

\section{References}

[1] International Union of Crystallography. Online Dictionary of Crystallography [Internet]. Available from: https://dictionary.iucr.org/Main_Page

[2] Chapuis G, Brock CP. The IUCr Online Dictionary of Crystallography. In International Union of Crystallography newsletter, Number 27. Available from: https://www.iucr.org/news/newsletter/volume-27/number-1/the-iucr-onlinedictionary-of-crystallography

[3] Study.com. Crystal: Definition, types, structure \& properties [Internet]. Available from: https://study.com/academy/lesson/crystal-definition-types-structureproperties.html

[4] McClure T. Dark crystals: the brutal reality behind a booming wellness craze. The Guardian. 2019 Sep 17. Available from: https://www.theguardian.com/lifeandstyle/2019/sep/17/healing-crystalswellnessmining-madagascar

[5] Cotton FA, Troup JM. Further exotic products from the reaction of diiron nonacarbonyl with bicyclo[6.2.0]deca-2,4,6-triene. X-ray crystallography as a practical means of cheap, rapid, and definitive analysis. J Am Chem Soc. 1973;95:3798-3799.

[6] Spek AL. CheckCIF validation ALERTS: What they mean and how to respond. Acta Cryst. 2020;E76:1-11. doi: 10.1107/S2056989019016244

[7] Spek AL. What makes a crystal structure report valid? Inorg Chim Acta. 2018;470:232-237. doi: 10.1016/j.ica.2017.04.036

[8] Fanwick PE. Crystallography for chemists. Newcastle upon Tyne: Cambridge Scholars Publishing; 2019. ISBN: 978-1-5275-3167-3.

[9] Girolami GS. X-Ray crystallography. Mill Valley, CA: University Science Books; 2016. ISBN: 978-1891389771.

[10] Clegg W, editor. Crystal structure analysis: principles and practice, 2nd edition. (International Union of Crystallography texts on crystallography - 13). Oxford: Oxford University Press; 2009. ISBN: 978-0199219476. 
[11] Sands DE. Vectors and tensors in crystallography. Reading, MA: Addison-Wesley Publishing Company; 1982. IBSN 0-201-07147-9.

[12] Lanczos C. Applied analysis. Englewood Cliffs, NJ: Prentice-Hall; 1956.

[13] Stout GH, Jensen LH. X-ray structure determination: A Practical Guide. New York: Macmillan; 1968.

[14] Borchardt-Ott W. Crystallography, 2nd ed. Gould RO, translator. Berlin: SpringerVerlag; 1995. ISBN 3-540-59478-7.

[15] Woolfson MM. An introduction to x-ray crystallography. Cambridge: Cambridge University Press; 1970. SBN 521074401.

[16] Dunitz JD. X-ray analysis and the structure of organic molecules. Ithaca NY: Cornell University Press; 1979. ISBN 0-8014-1115-7.

[17] Wilson AJC, editor. International tables for crystallography, Volume C: Mathematical, physical and chemical tables. Dordrecht: Kluwer Academic Publishers; 1992. Table 6.1.1.4 (scattering factors: pp. 500-502; dispersion corrections: Table 4.2.6.8, pp. 219-222; absorption corrections: Table 4.2.4.2, pp. 193-199).

[18] Macchi P. Modern charge density studies: the entanglement of experiment and theory. Cryst Rev. 2013;19:58-101. doi:10.1080/0889311X.2013.785538

[19] Macchi P, Gillet J-M, Taulelle F, Campo J, Claiser N, Lecomte C. Modelling the experimental electron density: only the synergy of various approaches can tackle the new challenges. IUCrJ. 2015;2:441-451. doi: 10.1107/S2052252515007538

[20] Capelli SC, Bürgi H-B, Dittrich B, Grabowsky S, Jayatilaka D. Hirshfeld atom refinement. IUCrJ. 2014;1:361-379. doi: 10.1107/S2052252514014845

[21] Hirshfeld FL. Bonded-atom fragments for describing molecular charge densities. Theor Chim Acta. 1977;44:129-138. doi: 10.1007/BF00549096

[22] Lübben J, Wandtke CM, Hübschle CB, Ruf M, Sheldrick GM, Dittrich B. Aspherical scattering factors for SHELXL - model, implementation and application. Acta Cryst. 2018;A75:50-62. doi: 10.1107/S2053273318013840.

[23] Azároff LV. Elements of x-ray crystallography. New York: McGraw-Hill; 1968.

[24] Massa W. Crystal structure determination. 2nd ed. Gould RO, translator. Berlin: Springer-Verlag; 2004. ISBN 3-540-20644-2.

[25] Buerger MJ. Elementary crystallography (paperback ed.). Cambridge, MA: MIT Press; 1978. (Original edition published by John Wiley and Sons, Inc., 1956.) ISBN 0-262-52048-6. 
[26] Bragg WH. The structure of organic crystals. Proc Phys Soc London 1921;34:3350.

[27] Dickinson RG, Raymond AL. The crystal structure of hexamethylenetetramine. J Am Chem Soc. 1923;45:22-29.

[28] Gonell HW, Mark H. Röntgenographische Bestimmung der Strukturformel des Hexamethylentetramins [X-ray determination of the structure of hexamethylenetetramine]. Z Phys Chem. 1923;107:181-218.

[29] Lonsdale K. The structure of the benzene ring in hexamethylbenzene. Proc R Soc A. 1929;123:494-515.

[30] Lonsdale K. X-ray evidence on the structure of the benzene nucleus. Trans Faraday Soc. $1929 ; 25: 352-366$.

[31] Desiraju GR. Crystal engineering: the design of organic solids, Amsterdam: Elsevier; 1989.

[32] Hamilton WC, Ibers JA. Hydrogen bonding in solids: Methods of molecular structure determination. New York: W. A. Benjamin; 1968.

[33] Mir NA, Dubey R, Desiraju GR. Strategy and methodology in the synthesis of multicomponent molecular solids: The quest for higher cocrystals. Acc Chem Res. 2019;52:2210-2220. doi: 10.1021/acs.accounts.9b00211

[34] Saccone M, Catalano L. Halogen bonding beyond crystals in materials science. J Phys Chem B. 2019;123:9281-9290. doi: 10.1021/acs.jpcb.9b07035

[35] Johnson CK. OR TEP: A FORTRAN thermal-ellipsoid plot program for crystal structure illustrations. ONRL report \#3794. Oak Ridge, TN: Oak Ridge National Laboratory; 1965.

[36] Burnett MN, Johnson CK. Ortep-III: Oak Ridge Thermal Ellipsoid Plot Program for Crystal Structure Illustrations. ORNL report \#6895. Oak Ridge, TN: Oak Ridge National Laboratory; 1996.

\section{Figure Captions}

Figure 1. (a) Different ways of defining a formally valid primitive unit mesh on a twodimensional net. (b) Fractional crystallographic coordinates xyz expressed in terms of a skew-angular reference frame.

Figure 2. Representation of the monoclinic space group $P 2$, extracted from the International Tables for Crystallography. 
Figure 3. (a) The basic elements of space group P2: a two-fold axis (set along $\boldsymbol{b}$ ) and a three-dimensional space lattice, with two lattice repeats perpendicular to the two-fold axis. Interaction of the two-fold symmetry element with translations perpendicular to it require the presence of interleaved two-fold symmetry axes (b) along the $\boldsymbol{a}$-direction, (c) along the $\boldsymbol{c}$-direction, and (d) along [101].

Figure 4. An improper two-fold symmetry element -- a mirror plane -- is accompanied by interleaved mirror planes at half-lattice repeats.

Figure 5. (a) The basic elements of a space group with four-fold symmetry: the fourfold axis and lattice translations perpendicular to it. (b) Closure requires the presence of an additional four-fold axis ('2' in red) passing through the center of the $\boldsymbol{a b}$-face of the cell. (c) The presence of two-fold symmetry interleaved along the $\boldsymbol{a}$ - and $\boldsymbol{b}$-axes is also required by closure.

Figure 6. (a) A three-fold rotation axis on a space lattice. (b) The further three-fold axes, '2' and '3' in red, required by closure.

Figure 7. Representation of a six-fold rotation axis on a space lattice. The symmetry element is accompanied by an entourage of three-fold and binary symmetry elements.

\section{Subject Index}

'Additional' symmetry 19-26

Addressable point 30

Adjoint reference frame 7-9

Asymmetric unit 21

Atom designator code 28-30

Atomic coordinates 8

Atomic displacement parameters 16-17

Atomic scattering factor 16-19

Bragg, W. H. 26-27

Buerger, M. J. 22-23

Calculation of interatomic distance 9-10

Closure 20

Convolution 6,14 
Convolution theorem 14

Convolution approximation 17

Cotton, F. A. 4

Crystal engineering 27-28

Delta function 6,13-15

Dunitz, J. D. 17

Fourier transform 6,14-15

Fractional crystallographic coordinates 6,7-8,

Lanczos, C. 9

Lattice 11-14

Massa, W. 21

Metric tensor 10

Online Dictionary of Crystallography 3

Reciprocal lattice 8

Representation of atoms in crystals 15-16, 29-30

Sands, D. E. 8

Skew-angular reference frame 7-11

Structure factor 14-15

Structure validation 5

Synthetic crystals 26-27

Troup, J. M. 4

Unit cell 11-12

Woolfson, M. M. 14 
Figure 1. (a) Different ways of defining a formally valid primitive unit mesh on a twodimensional net. (b) Fractional crystallographic coordinates xyz expressed in terms of a skew-angular reference frame.
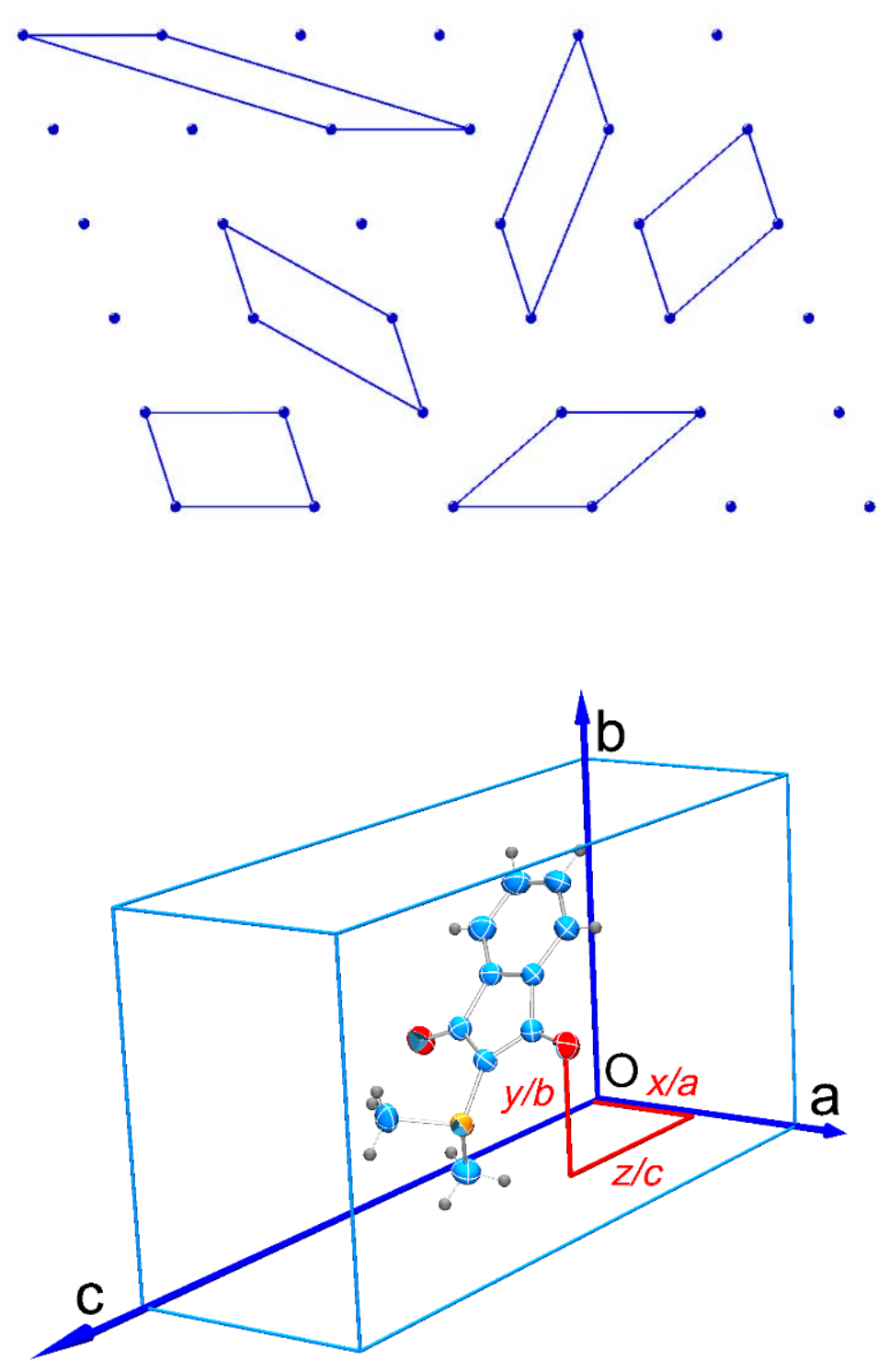
Figure 2. Representation of the monoclinic space group $P 2$, extracted from the International Tables for Crystallography.

$P 2$

$C_{2}^{1}$

$P 121$

No. 3

UNIQUE AXIS $b$
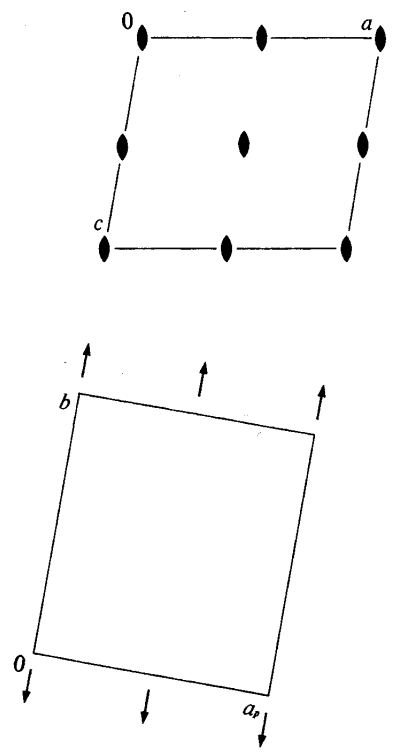

Generators selected $(1) ; t(1,0,0) ; t(0,1,0) ; t(0,0,1) ;(2)$

\section{Positions}

Multiplicity,

Wyckoff letter,

Site symmetry

2 e 1

(1) $x, y, z$

(2) $\bar{x}, y, \bar{z}$
2

Monoclinic

Patterson symmetry $P 12 / m 1$
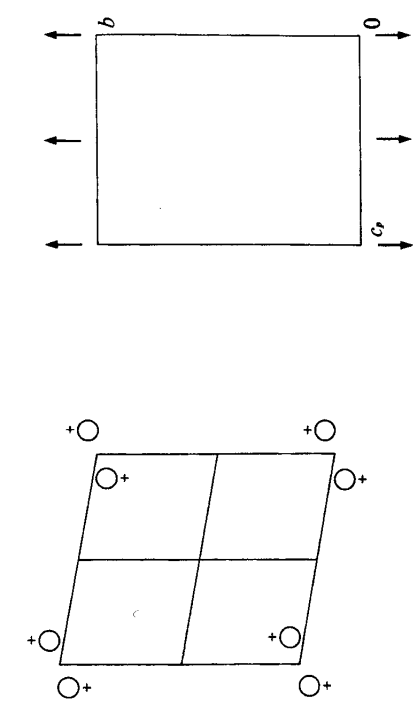

Reflection conditions

General:

no conditions

Special: no extra conditions 
Figure 3. (a) The basic elements of space group P2: a two-fold axis (set along $\boldsymbol{b}$ ) and a three-dimensional space lattice, with two lattice repeats perpendicular to the two-fold axis. Interaction of the two-fold symmetry element with translations perpendicular to it require the presence of interleaved two-fold symmetry axes (b) along the $\boldsymbol{a}$-direction, (c) along the $\boldsymbol{c}$-direction, and (d) along [101].
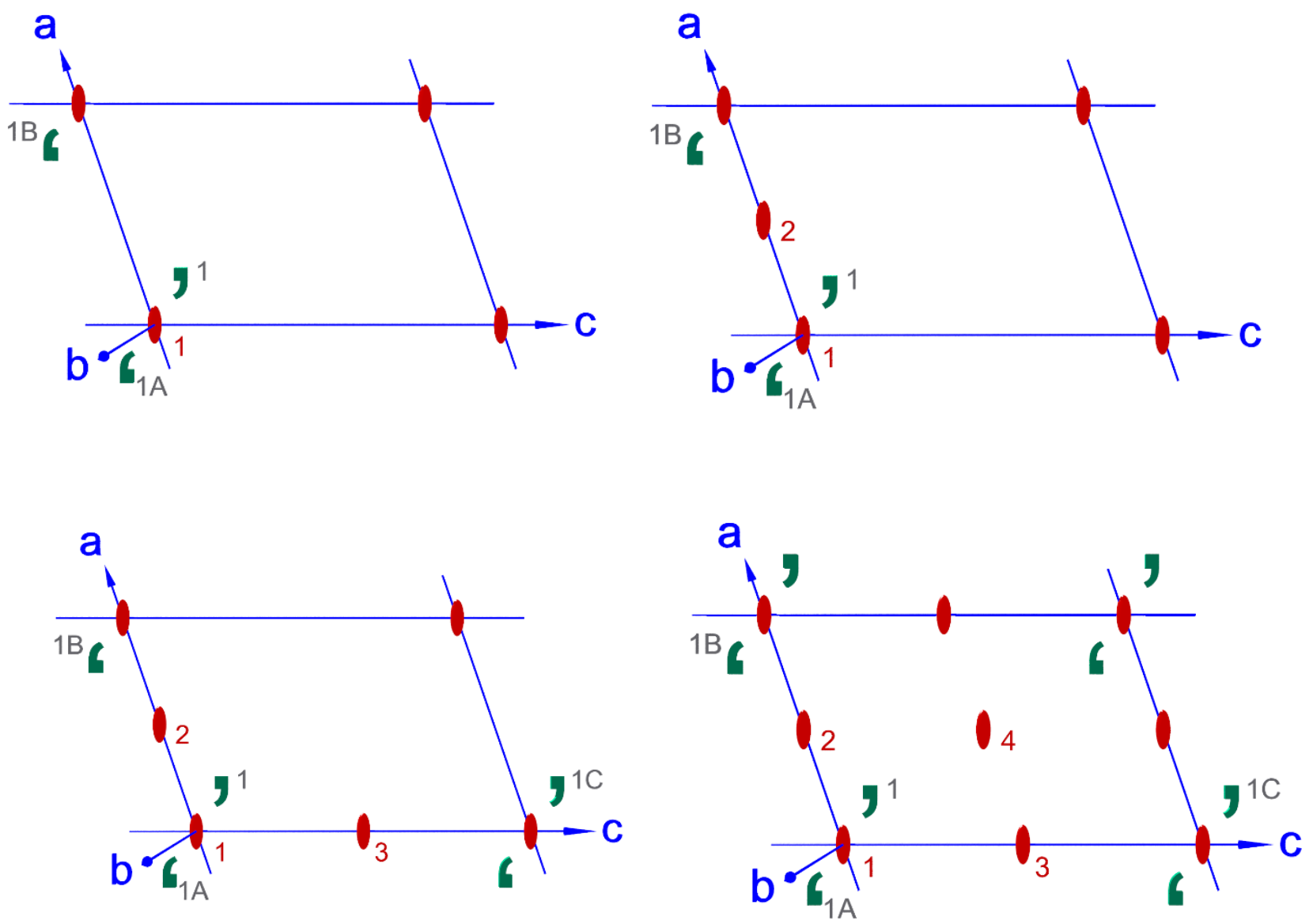
Figure 4. An improper two-fold symmetry element -- a mirror plane -- is accompanied by interleaved mirror planes at half-lattice repeats.

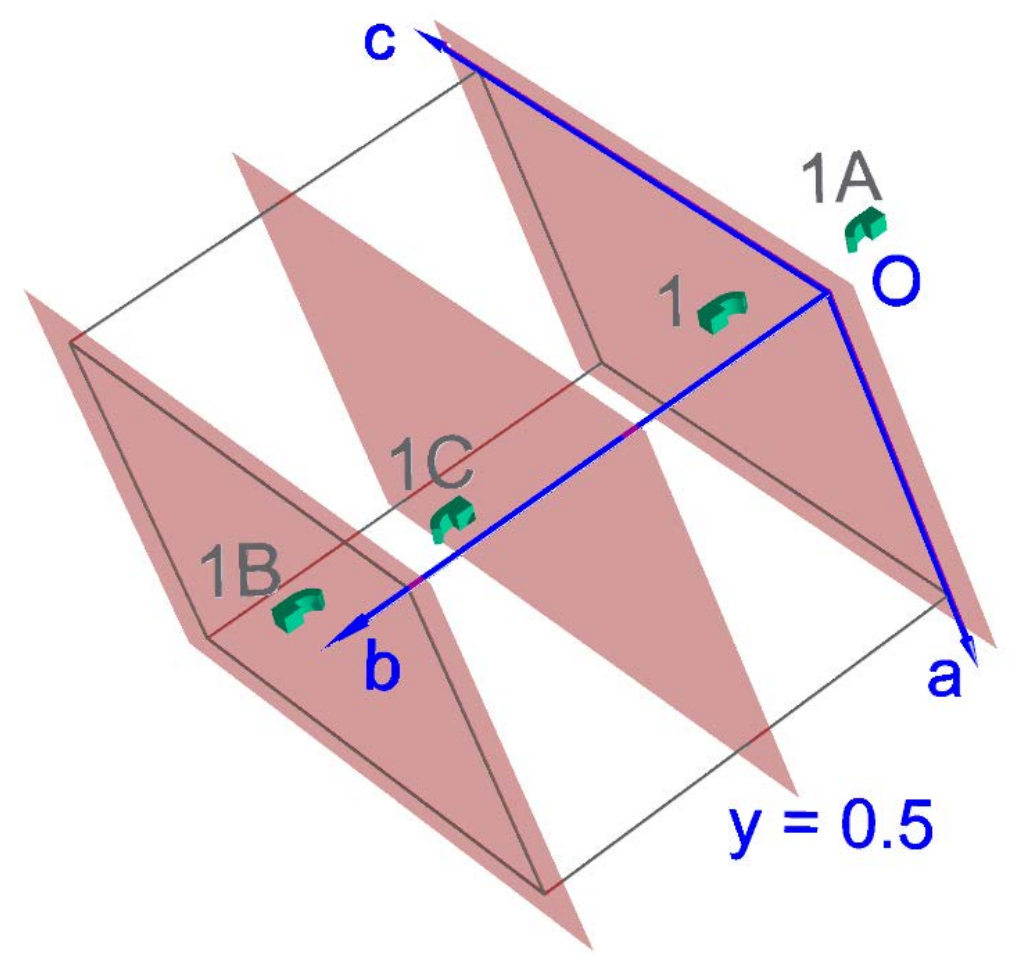


Figure 5. (a) The basic elements of a space group with four-fold symmetry: the fourfold axis and lattice translations perpendicular to it. (b) Closure requires the presence of an additional four-fold axis ('2' in red) passing through the center of the $\boldsymbol{a b}$-face of the cell. (c) The presence of two-fold symmetry interleaved along the $\boldsymbol{a}$ - and $\boldsymbol{b}$-axes is also required by closure.
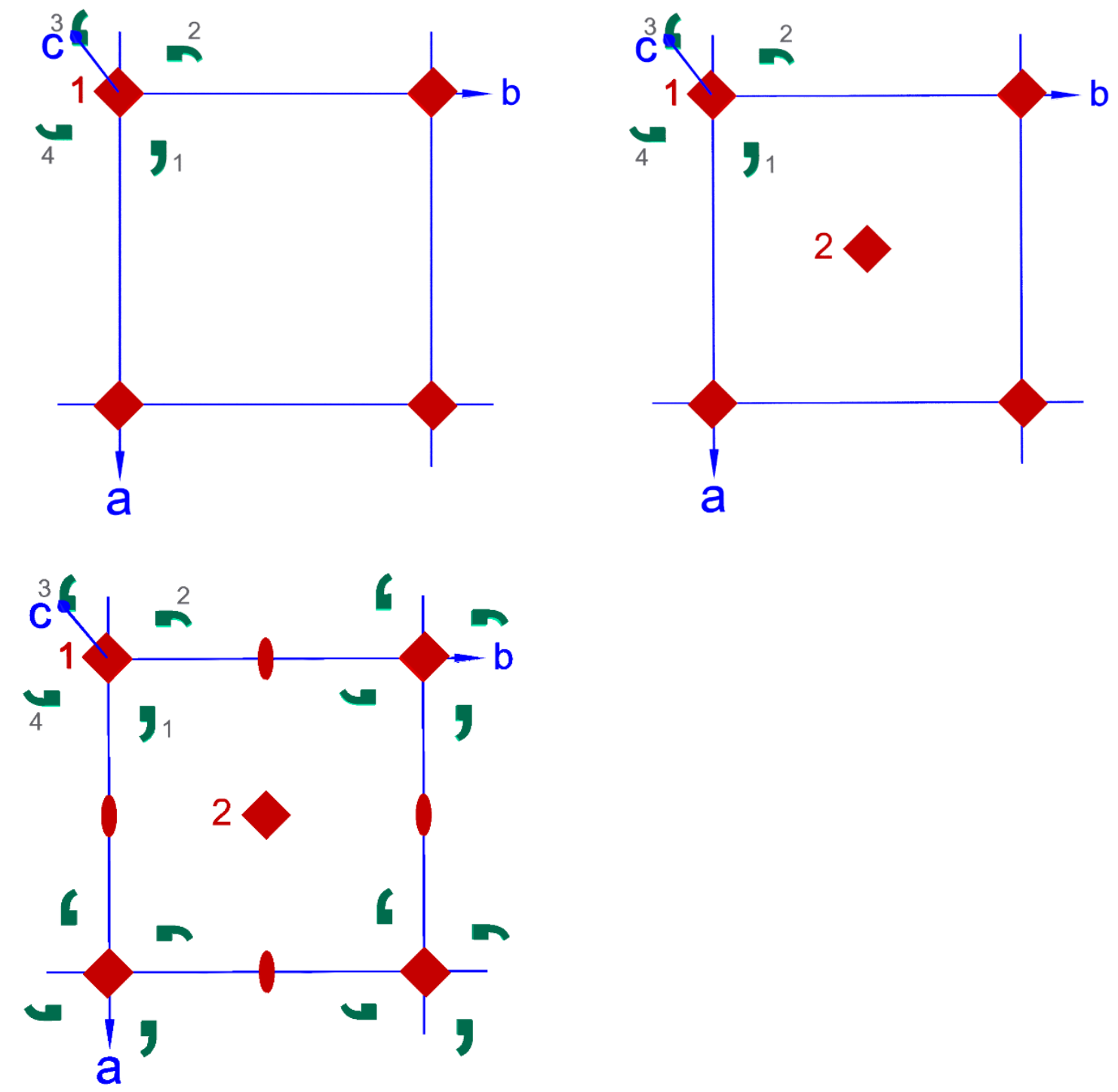
Figure 6. (a) A three-fold rotation axis on a space lattice. (b) The further three-fold axes, '2' and '3' in red, required by closure.
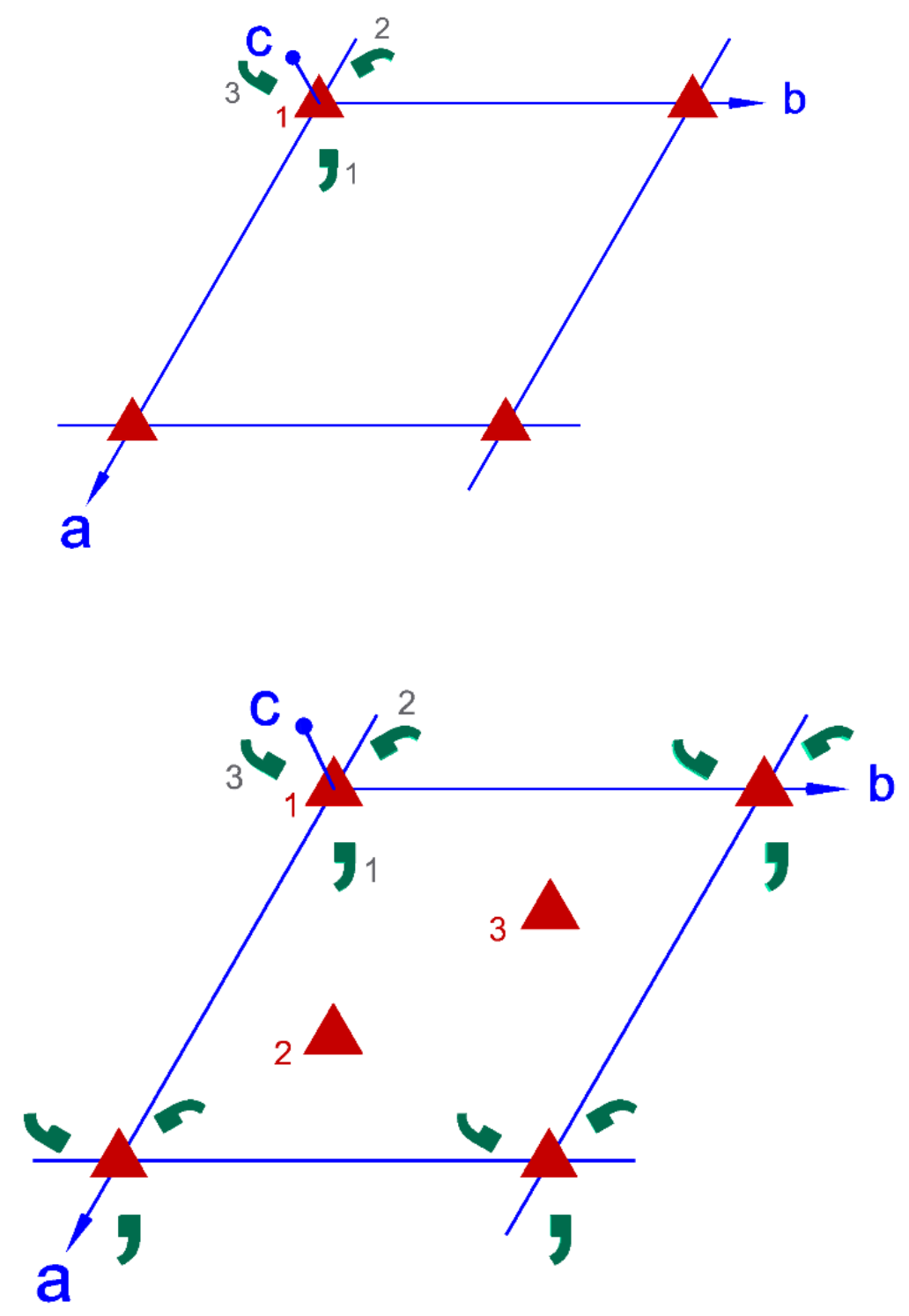
Figure 7. Representation of a six-fold rotation axis on a space lattice. The symmetry element is accompanied by an entourage of three-fold and binary symmetry elements.

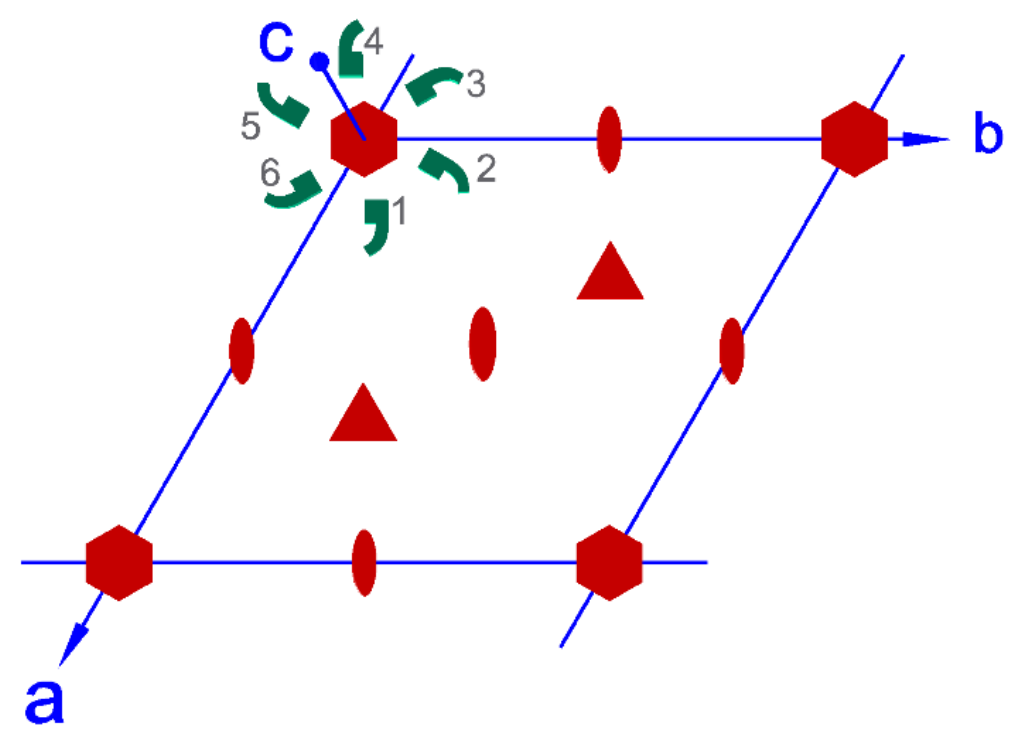

